Nervenarzt 2021 • 92:731-732

https://doi.org/10.1007/s00115-021-01149-2

Angenommen: 18. Mai 2021

(c) Springer Medizin Verlag $\mathrm{GmbH}$, ein Teil von Springer Nature 2021

\title{
Thrombektomie beim Schlaganfall - von klinischen Studien zur täglichen Praxis
}

\author{
Götz Thomalla · Jens Fiehler ${ }^{2}$ \\ ' Klinik und Poliklinik für Neurologie, Kopf- und Neurozentrum, Universitätsklinikum Hamburg-Eppendorf, \\ Hamburg, Deutschland \\ ${ }^{2}$ Klinik und Poliklinik für Neuroradiologische Diagnostik und Intervention, Zentrum für Radiologie und \\ Endoskopie, Universitätsklinikum Hamburg-Eppendorf, Hamburg, Deutschland
}

Die mechanische Thrombektomie hat die Behandlung des akuten Schlaganfalls revolutioniert. Patienten mit akutem Verschluss einer großen intrakraniellen Arterie, welche in früheren Jahren in der Mehrzahl große Infarkte mit nachfolgender schwerer Behinderung entwickelten, können bei rechtzeitiger endovaskulärer Behandlung inzwischen vielfach ohne relevante Einschränkungen das Krankenhaus verlassen. Die Basis für die Erfolgsgeschichte der Thrombektomie ist eine Serie randomisierter Studien, die den klaren Nachweis für den Nutzen der Thrombektomie erbracht haben [1, 2]. In der Folge wurden die bereits bestehenden Kapazitäten an interventioneller Schlaganfallbehandlung zu einem nahezu flächendeckenden Netz von Thrombektomiezentren ausgebaut [3, 4].

\section{I) Die gute Kooperation zwischen Neurologie und Neuroradiologie bestimmt den Behandlungserfolg}

Dabei ist die Akutbehandlung des Schlaganfalls ein dynamisches Feld. In den vergangenen Jahren brachten jedes Jahr neue klinische Studien neue Erkenntnis mit häufig direkten Auswirkungen auf die klinische Praxis. Das aktuelle Themenheft bietet ei-

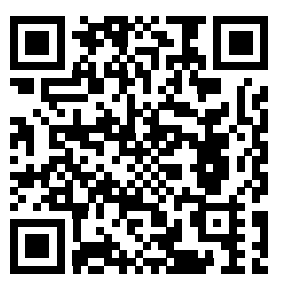

QR-Code scannen \& Beitrag online lesen ne Übersicht zu neuen Entwicklungen wie auch zu offenen Fragen zur Thrombektomie. Die hier gesammelten Artikel sollen Ihnen helfen, auf dem neuesten Stand der Entwicklung zu bleiben und die aktuellen Diskussionen zu verfolgen, die derzeit zu dem Themenfeld geführt werden.

Die Behandlung des akuten Schlaganfalls ist immer interdisziplinär, und insbesondere die gute Kooperation zwischen den zwei Disziplinen Neurologie und Neuroradiologie ist die Grundlage für eine gute Organisation der Behandlung. Aus diesem Grund freuen wir uns besonderes, dass wir für dieses Themenheft nicht nur ausgewiesene klinische und wissenschaftliche Experten/innen auf dem Gebiet der Thrombektomie gewinnen konnten, sondern dass jeder Beitrag von einem Tandem aus einem/r Neurologen/in und einem $/ r$ Neuroradiologen/in verfasst wurde.

Der letzte große Meilenstein in der Thrombektomie kam durch zwei klinische Studien, DAWN und DEFUSE-3, welche eindrücklich gezeigt haben, dass bei Verwendung erweiterter Bildgebung zur Patientenauswahl der Behandlungserfolg der Thrombektomie nicht an ein festes Zeitfenster gebunden ist $[5,6]$. Die verschiedenen Ansätze zur Bildgebung in den positiven Studien werfen dabei die Frage auf, wie die Patientenauswahl zur Thrombektomie in der klinischen Praxis erfolgen soll. Cheng und Broocks geben in ihrem Artikel eine Übersicht über die Daten hierzu, diskutieren Vor- und Nachteile der verschiedenen Ansätze und geben praktische Empfehlungen für die Auswahl der Bildgebung in der klinischen Routine.

\section{》) Registerdaten dokumentieren die Ergebnisse der Thrombektomie im klinischen Alltag}

Die häufig hoch selektierten Patientenpopulationen in klinischen Studien unterscheiden sich in vieler Hinsicht von den Patienten, bei denen wir im Alltag vor der Frage stehen, ob eine Thrombektomie indiziert ist. Beim Transfer von Studienergebnissen in die klinische Praxis stoßen wir daher immer wieder an Grenzen bzw. auf Fragen, die sich anhand der Studiendaten nicht beantworten lassen. Hier können Registerdaten helfen, die Ergebnisse der Thrombektomie im klinischen Alltag zu dokumentieren. In Deutschland wurde mit dem German Stroke Registry - Endovascular Treatment (GSR-ET) hier aus akademischem Antrieb und unabhängig von der Industrie ein einmaliges Register etabliert [7], welches inzwischen bereits Datensätze von mehr als 10.000 Thrombektomien enthält. Auch aus anderen Ländern gibt es vergleichbare Daten. In ihrem Artikel präsentieren Tiedt und Dorn eine Übersicht über Daten zur Thrombektomie aus der klinischen Praxis und zeigen auf, wie uns diese Daten helfen können, offene Fragen zu beantworten.

Die erste dokumentierte endovaskuläre Rekanalisationsbehandlung beim akuten Schlaganfall wurde vor mehr als 40 Jahren (Ende 1980) von Zeumer et al. im vertebrobasilären Stromgebiet durchgeführt, wenn auch lange vor der Ära der Thrombektomie mittels moderner Stentriever [8]. Leider sind bis heute die Daten aus klinischen Studien zur Thrombektomie im vertebro- 
basilären Stromgebiet limitiert. Auch die kürzlich präsentierten Daten der BASICSStudie haben hier mit ihren neutralen Ergebnissen nur begrenzt Abhilfe schaffen können und viele Fragen offen gelassen. Maegerlein und Pütz adressieren dieses Thema in ihrem Artikel und diskutieren die aktuellen Ergebnisse zur Thrombektomie bei Verschluss der A. basilaris und A. vertebralis.

Abschließend erörtern Psychogios und Kollegen die Grenzbereiche der Thrombektomie. Nach wie vor sind relevante Fragen zur Thrombektomie beim akuten Schlaganfall ungelöst, die Indikation zur Thrombektomie in Grenzfällen wie bei Patienten mit großer Infarktläsion, Patienten mit geringem klinischem Defizit oder peripheren Gefäßverschlüssen unklar. Laufende Studien werden hoffentlich in den kommenden Jahren hier weitere Klärung bringen.

Mit dieser Sammlung von Beiträgen bietet das Themenheft eine praktisch hoch relevante aktuelle Übersicht und kritische Diskussion der aktuellen Datenlage zur Thrombektomie beim Schlaganfall.

Eine interessante Lektüre wünschen

Götz Thomalla und Jens Fiehler

\section{Korrespondenzadresse}

\section{Prof. Dr. Götz Thomalla}

Klinik und Poliklinik für Neurologie, Kopf- und Neurozentrum, Universitätsklinikum HamburgEppendorf

Martinistr. 52, 20251 Hamburg, Deutschland thomalla@uke.de

\section{Prof. Dr. Jens Fiehler}

Klinik und Poliklinik für Neuroradiologische Diagnostik und Intervention, Zentrum für Radiologie und Endoskopie, Universitätsklinikum Hamburg-Eppendorf

Martinistr. 52, 20251 Hamburg, Deutschland fiehler@uke.de

Interessenkonflikt. G. Thomalla und J. Fiehler geben an, dass kein Interessenkonflikt besteht.

\section{Literatur}

1. Goyal M, Menon BK, van Zwam WH et al (2016) Endovascular thrombectomy after large-vessel ischaemic stroke: a meta-analysis of individual patient data from five randomised trials. Lancet 387(10029):1723-1731 (Apr)
2. Turc G, Bhogal P, Fischer U et al (2019) European Stroke Organisation (ESO)- European Society for Minimally Invasive Neurological Therapy (ESMINT) guidelines on mechanical thrombectomy in acute ischemic stroke. J Neurointerv Surg 11(6):535-538

3. Krogias C et al (2017) Verfügbarkeit der mechanischen Thrombektomie bei akutem Hirninfarkt. Nervenarzt 88(10):1177-1185

4. Rohde S, Weber W, Berlis A et al (2021) Acute endovascular stroke treatment in Germany in 2019: results from a nationwide database. Clin Neuroradiol 31(1):11-19

5. Albers GW, Marks MP, Kemp S et al (2018) Thrombectomy for stroke at 6 to 16 hours with selection by perfusion imaging. $\mathrm{N}$ Engl J Med 378:708-718

6. Nogueira RG, Jadhav AP, Haussen DC et al (2018) Thrombectomy 6 to 24 hours after stroke with a mismatch between deficit and infarct. N Engl J Med 378:11-21

7. Wollenweber FA, Tiedt S, Alegiani A et al (2019) Functional outcome following stroke thrombectomy in clinical practice. Stroke 50(9):2500-2506

8. Zeumer $\mathrm{H}$, Hacke $\mathrm{W}$, Kolmann $\mathrm{H}$, Poeck $\mathrm{K}$ (1982) Lokale Fibrinolysetherapie bei BasilarisThrombose. Dtsch Med Wochenschr 107:728-731

\section{Hilfestellungen für den Editorial Manager}

Das Einreichungs- und Begutachtungssystem Ihrer Zeitschrift

Sowohl für die ganz alltäglichen Fragen in der Handhabung des Editorial Managers als auch für spezielle Problematiken finden Sie auf www.springermedizin.de/editorialmanager eine Vielzahl an Handreichungen, die Ihnen die Arbeit als Gutachter*in, Autor*in oder Herausgeber*in erleichtern.

Über Videos, einseitige Schritt-für-SchrittAnleitungen oder ein umfangreiches Manual werden Sie durch die einzelnen Punkte geführt, wie:

- Wie reiche ich ein Manuskript ein?

- Wie finde ich passende Gutachter*innen?

- Wie lade ich Gutachter*innen ein?

- Wie nehme ich ein Gutachten an bzw. lehne es ab?

- Wo erkenne ich, in welchem Status ein Mansukript ist?

- Wie ändere ich meine persönlichen Informationen?

- Wo kann ich meinen Urlaub eintragen?

Zugang auch über QR-Code:

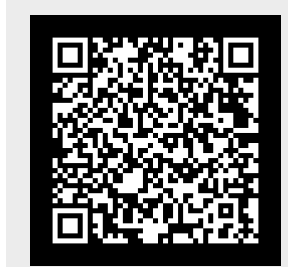

